\title{
Status of the UC-Berkeley SETI Efforts
}

\author{
E. J. Korpela ${ }^{a}$, D. P. Anderson ${ }^{a}$, R. Bankay ${ }^{a}$, J. Cobb ${ }^{a}$, A. Howard ${ }^{a}$, \\ M. Lebofsky ${ }^{a}$, A. P. V. Siemion ${ }^{a}$, J. von Korff ${ }^{b}$, and D. Werthimer ${ }^{a}$ \\ ${ }^{a}$ University of California, Berkeley, CA, 94720 USA; \\ ${ }^{b}$ Kansas State University, Manhattan, KS, 66502 USA
}

\begin{abstract}
We summarize radio and optical SETI programs based at the University of California, Berkeley.

The SEVENDIP optical pulse search looks for ns time scale pulses at visible wavelengths. It utilizes an automated 30 inch telescope, three ultra fast photo multiplier tubes and a coincidence detector. The target list includes F, G, K and M stars, globular cluster and galaxies.

The ongoing SERENDIP V.v sky survey searches for radio signals at the 300 meter Arecibo Observatory. The currently installed configuration supports 128 million channels over a $200 \mathrm{MHz}$ bandwidth with $\sim 1.6 \mathrm{~Hz}$ spectral resolution. Frequency stepping allows the spectrometer to cover the full $300 \mathrm{MHz}$ band of the Arecibo L-band receivers. The final configuration will allow data from all 14 receivers in the Arecibo L-band Focal Array to be monitored simultaneously with over 1.8 billion channels.

SETI@ home uses the desktop computers of volunteers to analyze over 160 TB of data at taken at Arecibo. Over 6 million volunteers have run SETI@home during its 10 year history. The SETI@home sky survey is 10 times more sensitive than SERENDIP V.v but it covers only a $2.5 \mathrm{MHz}$ band, centered on $1420 \mathrm{MHz}$. SETI@ home searches a much wider parameter space, including 14 octaves of signal bandwidth and 15 octaves of pulse period with Doppler drift corrections from $-100 \mathrm{~Hz} / \mathrm{s}$ to $+100 \mathrm{~Hz} / \mathrm{s}$. SETI@ home is being expanded to analyze data collected during observations of Kepler objects of interest in May 2011.

The Astropulse project is the first SETI search for $\mu$ s time scale pulses in the radio spectrum. Because short pulses are dispersed by the interstellar medium, and the amount of dispersion is unknown, Astropulse must search through 30,000 possible dispersions. Substantial computing power is required to conduct this search, so the project uses volunteers and their personal computers to carry out the computation (using distributed computing similar to SETI@ home).
\end{abstract}

Keywords: radio instrumentation, FPGA spectrometers, SETI, optical SETI, Search for Extraterrestrial Intelligence, volunteer computing, radio transients, optical transients

\section{INTRODUCTION}

At the University of California, Berkeley, we have been conducting five SETI searches that are roughly orthogonal to each other in search space. These five searches are summarized in Table 1.

The SERENDIP V.v sky survey covers a relatively broad range of radio frequencies, but not as sensitively as SETI@ home. The SETI@ home sky survey is more sensitive and examines a much wider variety of signal types than SERENDIP, but only covers a narrow band centered on the $21 \mathrm{~cm}$ Hydrogen line (a "magic frequency"). The Astropulse program is the first search for $\mu$ s time scale radio pulses. The SEVENDIP optical pulse search is sensitive to low duty cycle ultra-short pulses (eg: pulsed lasers).

We describe each of these programs below.

Email: korpela@ssl.berkeley.edu

Telephone: +1 510 643-6538

URL: http://setiathome.berkeley.edu/

Instruments, Methods, and Missions for Astrobiology XIV, edited by Richard B. Hoover, Paul C. W. Davies,

Gilbert V. Levin, Alexei Yu. Rozanov, Proc. of SPIE Vol. 8152, 815212 - (C) 2011 SPIE

CCC code: $0277-786 X / 11 / \$ 18 \cdot$ doi: $10.1117 / 12.894066$

Proc. of SPIE Vol. 8152 815212-1 


\begin{tabular}{lcll} 
Program Name & Timescale & Wavelength & $\Delta v / v$ \\
\hline \hline SERENDIP V.v & $1 \mathrm{~s}$ & radio & 0.21 \\
SETI@ home & $1 \mathrm{~ms}-10 \mathrm{~s}$ & radio & 0.0018 \\
Fly's Eye & $\mathrm{ms}$ & radio & 0.15 \\
Astropulse & $\mu \mathrm{s}$ & radio & 0.0018 \\
SEVENDIP & $\mathrm{ns}$ & optical & 0.8 \\
\hline
\end{tabular}

Table 1. SETI programs at the University of California, Berkeley

\section{OPTICAL SETI}

There is no clear wavelength choice for SETI. Microwave, IR and visible wavelengths all have advantages and disadvantages, depending on what factors another civilization might choose to optimize (power, size, bandwidth, and/or beam size). Although optical photons require more energy to generate than radio photons, optical beam sizes are typically much smaller, and directed interstellar communication links can be more efficient. ${ }^{1-3}$

\section{SEVENDIP}

The SEVENDIP (Search for Extraterrestrial Visible Emissions from Nearby Developed Populations) program at Berkeley searches for nanosecond time scale pulses, perhaps transmitted by a powerful pulsed laser operated by a distant civilization. The target list includes mostly nearby F, G, K and M stars, plus a few globular clusters and galaxies. The pulse search utilizes Berkeley's 0.8 meter automated telescope at Leuschner observatory and specialized instrumentation to detect short pulses. A similar instrument has been developed at Harvard University. ${ }^{4}$

The SEVENDIP instrument uses beam splitters to feed light from the telescope onto three high speed photomultiplier tubes. ${ }^{5}$ These tubes have a rise time of $0.7 \mathrm{~ns}$ and are sensitive to $300-700 \mathrm{~nm}$ wavelengths. The three signals are fed to high speed amplifiers, fast discriminators, and a coincidence detector. Three detectors are needed to reject "false alarms," which can be caused by radioactive decay and scintillation in the PMT glass, cosmic rays, and ion feedback. These false alarms can happen often in a single PMT, but almost never occur in three PMT's simultaneously.

The Leuschner pulse search has examined several thousand stars so far, each star for one minute or more. The experiment's sensitivity is $1.5 \times 10^{-17} \mathrm{~W} / \mathrm{m}^{2}$ for a $1 \mathrm{~ns}$ pulse, which corresponds to $1.5 \times 10^{-28} \mathrm{~W} / \mathrm{m}^{2}$ average power if the pulse duty cycle is one nanosecond every 100 seconds.

\section{THE SERENDIP V.v ARECIBO SKY SURVEY}

The SERENDIP SETI program began 25 years ago; it has gone through four generations of instrumentation and has observed on 14 radio telescopes. During these twenty five years, SERENDIP's sensitivity has improved by a factor of ten thousand and the number of channels has increased from one hundred to more than one hundred million. ${ }^{6,7}$

The latest SERENDIP sky survey, SERENDIP V.v, began in earnest in 2009. Observations are ongoing. The survey utilizes the National Astronomy and Ionospheric Center's 305 meter radio telescope in Arecibo, Puerto Rico. The survey thoroughly covers $25 \%$ of the sky (declinations from $-2^{\circ}$ to $+38^{\circ}$ ). Each of the 10 million beams will be observed an average of three times during the five year survey. Multiple observations are needed because sources may scintillate ${ }^{8}$ or have short duty cycles, and many of our robust detection algorithms require multiple detections.

The sky survey utilizes real time 128 million channel FFT spectrum analyzers to search for narrow band radio signals in a $300 \mathrm{MHz}$ band centered at the $21 \mathrm{~cm}$ Hydrogen line $(1420 \mathrm{MHz})$. The currently installed system consists of one such instrument. We are working towards a final configuration consisting of 14 of these instruments, which will allow simultaneous analysis of data from all of the 14 receivers of the Arecibo L-band Focal Array (ALFA). The system has a 0.6 second integration time, $1.6 \mathrm{~Hz}$ channel width, and a sensitivity of $10^{-24} \mathrm{~W} / \mathrm{m}^{2}$.

SERENDIP V.v conducts observations continuously whenever the ALFA array is in use for other astronomical observations. SERENDIP data analysis is described by Cobb. ${ }^{9}$ Information on signals whose power exceeds 16 times the mean noise power are logged along with baseline power, telescope coordinates, time and frequency. This data is transmitted to Berkeley in real time; then, radio frequency interference (RFI) rejection algorithms are applied to the data, off-line, at UC 
Berkeley. After the RFI is rejected, computers search for candidate signals. SERENDIP's candidate detection algorithms are sensitive to several types of signals, which, individually or combined, may trigger an event to be noted for further study. These algorithms test for beam pattern matching, linear drift rates, regularly spaced pulses, multiple frequencies (particularly those periodic in frequency), and coincidence with nearby stars, globular clusters, or extra-solar planetary systems. Every few months, the entire data base is scanned for multiple detections - "signals" that are detected again when the telescope revisits the same sky coordinates. We test how well these multiple detections fit a barycentric reference frame. We also apply another test that allows much higher frequency separation, which is necessary if transmitters are not corrected for their planet's rotation and revolution. Data are simultaneously sent to Cornell University for analysis using other techniques.

Potential candidates are scored and ranked by the probability of noise causing that particular detection. In cases where multiple detections have been made, a joint probability is assessed. These joint probabilities are used for comparing candidates against each other and generating a prioritized candidate list for re-observation.

\section{THE SETI@HOME SKY SURVEY}

SETI@home data comes from the same piggyback receiver that SERENDIP uses at the Arecibo radio telescope. Whereas SERENDIP analyzes this data primarily using a special-purpose spectrum analyzer and supercomputer located at the telescope, SETI@home records the data, and then distributes the data through the Internet to hundreds of thousands of personal computers. This approach provides a tremendous amount of computing power, but limits the amount of data that can be handled. Hence SETI@ home covers a relatively narrow frequency range (2.5 MHz), but searches for a wider range of signal types, and with improved sensitivity. ${ }^{10,11}$

SETI@ @ome was launched on May 17, 1999. In its 10 years of operation, it has attracted over 5 million participants. SETI@ home is one of the largest supercomputers on our planet, currently averaging 3.5 PFLOP actual performance. Users are located in 226 countries, and about 50\% of the users are from outside of the United States.

Although SETI@home has 1/80 the frequency coverage of SERENDIP V.v, its sensitivity is roughly ten times better. The SETI@ @ome search also covers a much richer variety of signal bandwidths, drift rates, and time scales than SERENDIP V.v or any other SETI program to date.

Primary data analysis, done using distributed computing, computes power spectra and searches for "candidate" signals such as spikes, Gaussians, and pulses. Secondary analysis, done on the project's own computers, rejects RFI and searches for repeated events within the database of candidate signals.

SETI@ home covers a $2.5 \mathrm{MHz}$ bandwidth centered at the $1420 \mathrm{MHz}$ Hydrogen line from each of the 14 ALFA receivers (7 beams $\times 2$ polarizations). The $2.5 \mathrm{MHz}$ band is recorded continuously onto SATA disks with two bits per complex sample. Disks are mailed to UC Berkeley for analysis.

SETI@home data disks from the Arecibo telescope are divided into small "work units" as follows: the $2.5 \mathrm{MHz}$ bandwidth data is first divided into 256 sub-bands; each work unit consists of 107 seconds of data from a given $9,765 \mathrm{~Hz}$ sub-band. Work units are then sent over the Internet to the client programs for the primary data analysis.

Because an extraterrestrial civilization's signal has unknown bandwidth and time scale, the client software searches for signals at 15 octave spaced bandwidths ranging from $0.075 \mathrm{~Hz}$ to $1220 \mathrm{~Hz}$, and time scales from $0.8 \mathrm{~ms}$ to 13.4 seconds. The rest frame of the transmitter is also unknown (it may be on a planet that is rotating and revolving), so extraterrestrial signals are likely to be drifting in frequency with respect to the observatory's topocentric reference frame. Because the reference frame is unknown, the client software examines about 1200 different Doppler acceleration frames (dubbed "chirp rates"), ranging from $-100 \mathrm{~Hz} / \mathrm{sec}$ to $+100 \mathrm{~Hz} / \mathrm{sec}$.

At each chirp rate, peak searching is done by computing non-overlapping FFTs and their resulting power spectra. FFT lengths range from 8 to 131,072 in 15 octave steps. Peaks greater than 24 times the mean power are recorded and sent back to the SETI@ home server for further analysis.

Besides searching for peaks in the multi-spectral-resolution data, SETI@home also searches for signals that match the telescope's Gaussian beam pattern. Gaussian beam fitting is computed at every frequency and every chirp rate at spectral resolutions ranging from 0.6 to $1220 \mathrm{~Hz}$ (temporal resolutions from $0.8 \mathrm{~ms}$ to 1.7 seconds). The beam fitting algorithm attempts to fit a Gaussian curve at each time and frequency in the multi-resolution spectral data. Gaussian fits whose power exceeds the mean noise power by a factor of 3.2 and whose reduced $\chi^{2}$ of the Gaussian fit is less than 1.42 are reported 
to the SETI@ @ome servers. SETI@ @ome also searches for pulsed signals using a modified Fast Folding Algorithm ${ }^{12}$ and an algorithm which searches for three regularly-spaced pulses. More details of the SETI@ home analysis can be found in Korpela et al. (2001) ${ }^{13}$ and Korpela (2011). ${ }^{14}$

In the near future, we will be adding the ability to detect autocorrelated signals. Harp et al. ${ }^{15}$ propose that an extraterrestrial civilization could send a beacon that contains information (has appreciable bandwidth), yet is easily detectable. This could be done by sending a signal and then, after a short delay, starting the broadcast of a copy of the signal. A signal of this type can be detected through autocorrelation, which will show a peak power at the given delay. Once the delay is known, the information within the signal can, in principle, be recovered. A version of SETI@ home containing an autocorrelation detector, acting on delays up to 13.4 seconds, is being beta tested, and should be released in late summer of 2011.

To determine signals of interest the data for each sky position which has recently received new potential signals is examined by our Near-Time Persistency Checker (NTPCkr). This program scores candidates based upon the probability that the assemblage of potential signals seen could be due to random fluctuations in the noise background. This score includes the probability of multiple detections in any reference frame, the probability of repeated detection in the barycentric frame, the goodness of fit with the antenna beam pattern, and conicidence with known planets, nearby stars (from the Hipparcos catalog) or galaxies. We generate a ranked list of our best candidates for reobservation. ${ }^{16}$

Most of the signals found by the client programs turn out to be terrestrial based radio frequency interference (RFI). We employ a substantial number of algorithms to reject the several types of RFI ${ }^{9}$ from the best signals. Once RFI rejection has been performed on a candidate group, it is re-scored by the NTPCkr. ${ }^{17}$

\section{SETI@HOME-GREEN BANK}

In May of 2011, we utilized the 100m NRAO Green Bank Telescope (GBT) to observe 86 of the best planetary system candidates found by the Kepler mission up to that point. We selected distinct Kepler Input Catalog stars having either a Kepler Object of Interest (KOI) with a calculated equilibrium temperature between 230 and $380 \mathrm{~K}$, at least 4 KOIs or a KOI with inferred radius $<3.0$ Earth radii and an orbital period $>50$ days.

Using the GUPPI pulsar processor and modified software we were able to record eight $100 \mathrm{MHz}$ bands in dual polarization using 4 bits per complex sample, to provide simultaneous coverage between 1.1 and $1.9 \mathrm{GHz}$. Following the observations of individual candidates for 450 seconds each, we performed a 12 hour survey of the entire Kepler field. In addition to being analyzed using local computing clusters, we will be sending this data out to SETI@ home volunteers for analysis with the SETI@ home and Astropulse applications. Following some minor modifications to the SETI@ home and Astropulse software necessary to support the new data parameters, we expect to start sending this data out to volunteers starting in the fall of 2011.

\section{BOINC}

SETI@ home has clearly shown the viability of volunteer based distributed computing for other scientific problems. To this end we have developed an infrastructure dubbed BOINC (Berkeley Open Infrastructure for Network Computing), which can be used for other applications. ${ }^{18}$ The availability of this open source infrastructure has eased the development of other distributed applications. Our open source distributed computing infrastructure engages the public in climate modeling/global warming studies, (climatePrediction.net), HIV, malaria and cancer drug research (World Community Grid), particle physics (LHC@ @ome), gravity waves (Einstein@ home), protein structure (Rosetta@home), and of course SETI@ home. More than 50 distributed computing projects use this infrastructure.

The BOINC infrastructure has many advantages over the stand alone SETI@ home infrastructure. Volunteers can sign up for many projects and divide their computing resources among them. It is our hope that this will lead to a larger shared volunteer base. The BOINC infrastructure makes it possible for a project to include multiple separate applications and to distribute updates to applications without requiring complex user intervention. The SETI@ home BOINC "project" can include multiple "applications" to analyze data in different ways and to analyze data from different sources. Currently we distribute the standard SETI@ home application and the Astropulse application to users who have signed up for SETI@home. 
The BOINC client performs the communications necessary to download the SETI@ home application, to download the data to be analyzed, and to upload the results. It also can display screen saver graphics showing the status of the analysis. BOINC and SETI@ @ome are available for MacOS X, Windows and Linux in both 32 and 64 bit versions. Participants using other operating systems can download the source code, and can compile their own versions of BOINC and SETI@ home. Participants can download the client software at: http://setiathome.berkeley.edu/

\section{FLY'S EYE}

Our now completed "Fly's Eye" experiment searched for bright radio transient pulses at the Allen Telescope Array (ATA). The Allen Telescope Array has several advantages over other telescopes worldwide for performing transient searches, particularly when the search is for bright pulses. The ATA has 42 independently-steerable dishes, each $6 \mathrm{~m}$ in diameter. The beam size for individual ATA dishes is considerably larger than that for most other telescopes, such as VLA, NRAO Green Bank, Parkes, Arecibo, Westerbork and Effelsberg. By pointing each dish in a different direction the ATA can instantaneously observe a far larger portion of the sky than is possible with other telescopes. Conversely, when using the ATA dishes independently, the sensitivity of the ATA is far lower than that of other telescopes. The Fly's Eye instrument was built to search for bright radio pulses of submillisecond duration at the ATA. The instrument consisted of 44 independent spectrometers each processing a bandwidth of $210 \mathrm{MHz}$, and producing 128 -channel power spectrum at a rate of $1600 \mathrm{~Hz}$. Therefore each spectrum represents time domain data of length $0.625 \mathrm{~ms}$, and hence pulses as short as $0.625 \mathrm{~ms}$ can be resolved

Because interstellar pulses are dispersed by ionized interstellar gas, a pulse search involves correcting for this dispersion. The analysis required for the Fly's Eye experiment is, in principle, fairly simple. We wish to search over a wide range of dispersion measures to find large individual pulses. Specifically our processing requires that all the data be dedispersed with dispersion measures ranging from $50 \mathrm{pc} \mathrm{cm}^{-3}$ to $2000 \mathrm{pc} \mathrm{cm}^{-3}$. At each dispersion measure the data needs to be searched for 'bright' pulses. The processing chain is in practice significantly more complicated than this description suggests. Processing is performed on compute clusters, with input data formatted, divided and assigned to worker nodes for processing. In the worker node flow, the data is equalized, RFI rejection is performed, and finally a pulse search is performed through the range of dispersion measures. The results are written to a database where they can be subsequently queried. The key feature of the results is a table that lists, in order of decreasing significance, the pulses that were found and their dispersion measures.

We have been able to observe $150 \mathrm{deg}^{2}$ over 450 hours. We have successfully detected three pulsars (B0329+54, B0355+54, B0950+08) and six giant pulses from the Crab pulsar. We have not detected any other convincing bursts of astronomical origin in our survey data implying a limiting rate of less than $2 \mathrm{sky}^{-1}$ hour $^{-1}$ for $10 \mathrm{~ms}$ duration pulses having average apparent flux densities greater than $44 \mathrm{Jy}$. More information about "Fly's Eye" is available in Siemion et al. $(2010,2011)^{19,20}$

\section{ASTROPULSE}

Radio SETI searches to date have concentrated on narrow-band signals as opposed to wide-band signals such as pulses. The Astropulse project was the first SETI search for $\mu$ s radio pulses. Astropulse detects pulse widths ranging from $1 \mu$ s to $1 \mathrm{~ms}$. Such pulses might come from extraterrestrial civilizations, evaporating black holes, ${ }^{21,22}$ gamma ray bursts, certain supernovae, or pulsars. ${ }^{23}$ The Astropulse program mines the SETI@ home data archive for serendipitous detections of such events.

One of the unique features of this search is that it is the first pulse search to use coherent dedispersion in a "blind" fashion - we have no previous knowledge of a specific dispersion measure (DM) to examine. The reason this search has never been attempted before is due to the enormous computing power required.

The computing problem is eminently parallel in nature. Similar to SETI@ home, Astropulse uses volunteers and their personal computers to carry out the computation. Astropulse uses the general purpose distributed computing system we have developed (BOINC).

Thus far, Astropulse has examined several years of SETI@home data, resulting in more than 125 million potential detections. We are currently working on RFI rejection techniques to clean the data set of RFI, primarily due to aviation radars. Most RFI sources appear to have a stable dispersion measure. If we detect a pulse with the same dispersion measure, 


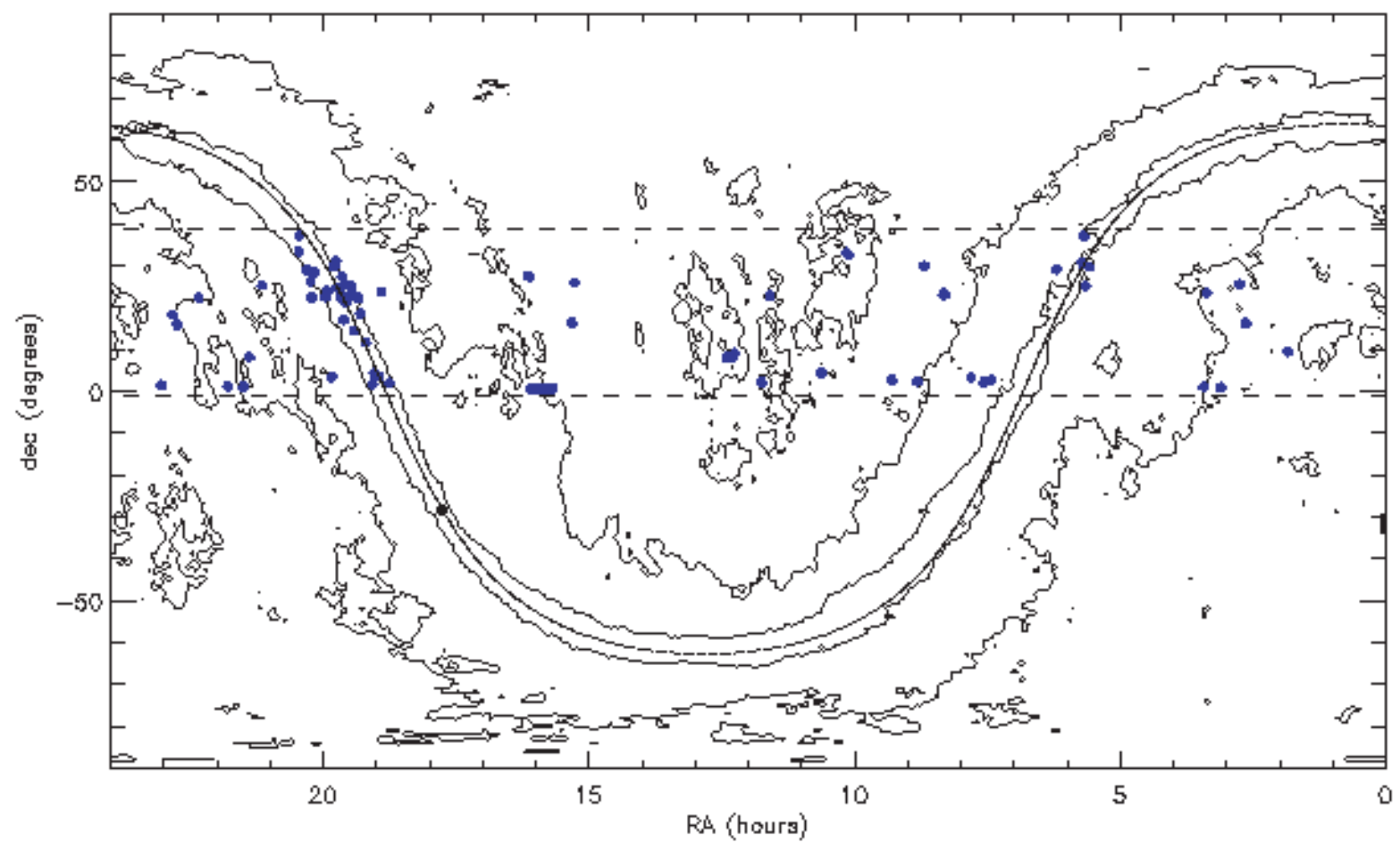

Figure 1. Distribution of Astropulse candidates on the sky. The dots are the potential sources. Contours are LAB HI survey ${ }^{26}$ logarithmic contours. The dashed horizontal lines represent the limits of Arecibo's viewing range.

but from a different part of the sky minutes to hours later, it is likely to be RFI. In practice we compute the likelihood of such coincidence happening and if that likelihood is below a threshold we conclude that it is RFI. We use two more steps to further select the best pulses. First we check to see that the pulse is truly broadband. Some pulses can be due to chance coincidence of peaks at two frequencies with a time offset. We eliminate these by checking the distribution of power vs frequency and using a $\chi^{2}$ test from a fit with a flat spectrum to determine whether it is a broad band pulse. Finally, because we have two polarizations being analyzed independently we can, at the cost of some sensitivity, require that the pulse be detected in both polarization.

Using this procedure on the first 12 million pulses we found, 330 pulses from 114 sky locations passed the test. Two of these locations correspond with known pulsars, leaving 112 that do not. These candidates are concentrated near the Galactic plane, much more than our observing time has been. A statistical analysis ${ }^{24}$ indicates a greater than $4 \sigma$ probability that this distribution is not due to chance. We reobserved most of these candidate locations in July 2011. However, given the volume of data, our analysis of these reobservations is just beginning.

More details of Astropulse can be found in von Korff (2010) ${ }^{24}$ and von Korff et al (2011). ${ }^{25}$

\section{ACKNOWLEDGMENTS}

This work has been supported by the Planetary Society, the SETI Institute, the University of California, Sun Microsystems and donations from individuals around the planet. Key hardware was donated by Network Appliance, Xilinx, Fujifilm Computers, Toshiba, Quantum, Hewlett Packard, and Intel Corp. We receive excellent support from the staff of the Arecibo Observatory, a part of that National Astronomy and Ionosphere Center, which is operated by Cornell University under a cooperative agreement with the National Science Foundation. We would also like to thank the Allen Telescope Array, a facility of the SETI Institute. The National Radio Astronomy Observatory in Green Bank, WV is a facility of the National Science Foundation operated under cooperative agreement by Associated Universities, Inc. This work has been supported in part by NSF Grants AST-0808175 and AST-0307956, and NASA Grant NNX09AN69. 


\section{REFERENCES}

[1] Lampton, M., "Optical SETI: The Next Search Frontier," in [Bioastronomy 99: A New Era in Bioastronomy], G. Lemarchand \& K. Meech, ed., ASP Conf. Ser. 213, 565- (2000) ADS: 2000ASPC. .213 . .565L .

[2] Schwartz, R. N. and Townes, C. H., "Interstellar and Interplanetary Communication by Optical Masers," Nature 190, 205-208 (Apr. 1961) DOI:10.1038/190205a0 .

[3] Townes, C. H., "Optical and infrared SETI," in [Astronomical and Biochemical Origins and the Search for Life in the Universe ], C. Batalli Cosmovici, S. Bowyer, \& D. Werthimer, ed., IAU Colloq. 161, 585- (Jan. 1997) ADS:1997abos.conf. . 585T .

[4] Howard, A., Horowitz, P., Coldwell, C., Latham, D., Papaliolios, C., Stefanik, R., Wolff, J., and Zajac, J., "Optical SETI at Harvard-Smithsonian," in [Bioastronomy 99: A New Era in Bioastronomy], G. Lemarchand \& K. Meech, ed., ASP Conf. Ser. 213, 545- (2000) ADS: 2000ASPC. .213 . .545H .

[5] Wright, S. A., Drake, F., Stone, R. P., Treffers, D., and Werthimer, D., "Improved optical SETI detector," in [The Search for Extraterrestrial Intelligence (SETI) in the Optical Spectrum III], S. A. Kingsley \& R. Bhathal, ed., Proc. SPIE 4273, 173-177 (Aug. 2001) DOI: 10.1117/12.435376 .

[6] Werthimer, D., Bowyer, S., Ng, D., Donnelly, C., Cobb, J., Lampton, M., and Airieau, S., "The Berkeley SETI program - SERENDIP IV instrumentation," in [Astronomical and Biochemical Origins and the Search for Life in the Universe ], C. Batalli Cosmovici, S. Bowyer, \& D. Werthimer, ed., IAU Colloq. 161, 683- (Jan. 1997) ADS:1997abos.conf . .683W .

[7] Bowyer, S., Werthimer, D., Donnelly, C., Cobb, J., Ng, D., and Lampton, M., "Twenty years of SERENDIP, the Berkeley SETI effort - Past results and future plans," in [Astronomical and Biochemical Origins and the Search for Life in the Universe ], C. Batalli Cosmovici, S. Bowyer, \& D. Werthimer, ed., IAU Colloq. 161, 667- (Jan. 1997) ADS:1997abos.conf . .667B .

[8] Cordes, J., Lazio, T., and Sagan, C., "Scintillation-Induced Intermittency in SETI," Astrophys. J. 487, 782- (1997) DOI:10.1086/304620.

[9] Cobb, J., Lebofsky, M., Werthimer, D., and Bowyer, S., "SERENDIP IV: Data Acquisition, Reduction and Analysis," in [Bioastronomy 99: A New Era in Bioastronomy], G. Lemarchand \& K. Meech, ed., ASP Conf. Ser. 213, 485(2000) ADS:2000ASPC. .213 . .485C .

[10] Anderson, D. P., Werthimer, D., Cobb, J., Korpela, E., and Lebofsky, M., "SETI@ home: Internet Distributed Computing for SETI," in [Bioastronomy 99: A New Era in Bioastronomy], G. Lemarchand \& K. Meech, ed., ASP Conf. Ser. 213, 511-(2000) ADS: 2000ASPC. .213..511A.

[11] Sullivan, W. T., Werthimer, D., Bowyer, S., Cobb, J., Gedye, D., and Anderson, D., "A new major SETI project based on Project SERENDIP data and 100,000 personal computers," in [Astronomical and Biochemical Origins and the Search for Life in the Universe ], C. Batalli Cosmovici, S. Bowyer, \& D. Werthimer, ed., IAU Colloq. 161, 729(Jan. 1997) ADS:1997abos.conf..729. .

[12] Staelin, D., "Fast folding algorithm for detection of periodic pulse trains," Proc. IEEE 57, 724-725 (Apr. 1969) DOI:10.1109/PROC.1969.7051.

[13] Korpela, E., Werthimer, D., Anderson, D., Cobb, J., and Leboisky, M., "SETI@ home-Massively Distributed Computing for SETI," Computing in Science and Engineering 3, 78-83 (Jan/Feb 2001) DOI: 10.1109/5992.895191

[14] Korpela, E. J., "Distributed Processing of SETI Data," in [Searching for Extraterrestrial Intelligence: SETI Past, Present, and Future (The Frontiers Collection)], Shuch, H. P., ed., 183-200, Springer, Heigelberg, DE (2011) DOI:10.1007/978-3-642-13196-7.

[15] Harp, G. R., Ackerman, R. F., Blair, S. K., Arbunich, J., Backus, P. R., Tarter, J. C., and the ATA Team, “A New Class of SETI Beacons That Contain Information," in [Communication with Extraterrestrial Intelligence (CETI)], Vakoch, D. A., ed., 37-44, SUNY Press, Albany, NY, USA (Apr. 2011).

[16] Korpela, E. J., Cobb, J., Fulton, S., Lebofsky, M., Heien, E., Person, E., Demorest, P., Bankay, R., Anderson, D., and Werthimer, D., "Three Years of SETI@ home: A Status Report," in [Bioastronomy 2002: Life Among the Stars], R. Norris \& F. Stootman, ed., IAU Symp. 213, 419-422 (June 2004) ADS: 2004 IAUS . .213 . .419K .

[17] Korpela, E. J., Cobb, J., Lebofsky, M., Siemion, A., von Korff, J., Bankay, R. C., Werthimer, D., and Anderson, D., "Candidate Identification and Interference Removal in SETI@ home," in [Communication with Extraterrestrial Intelligence (CETI)], Vakoch, D. A., ed., 37-44, SUNY Press, Albany, NY, USA (Apr. 2011). 
[18] Anderson, David P., "BOINC: A System for Public-Resource Computing and Storage," in [Proceedings of the 5th IEEE/ACM International Workshop on Grid Computing], GRID '04, 4-10, IEEE Computer Society, Washington, DC, USA (2004) DOI:10.1109/GRID.2004.14.

[19] Siemion, A., von Korff, J., McMahon, P., Korpela, E., Werthimer, D., Anderson, D., Bower, G., Cobb, J., Foster, G., Lebofsky, M., van Leeuwen, J., and Wagner, M., "New SETI sky surveys for radio pulses," Acta Astronautica 67, 1342-1349 (Dec. 2010) ADS:2010AcAau. .67.1342S.

[20] Siemion, A. P. V., Bower, G. C., Foster, G., McMahon, P. L., Wagner, M. I., Werthimer, D., Backer, D., Cordes, J., and van Leeuwen, J., "The Allen Telescope Array Fly's Eye Survey for Fast Radio Transients," submitted to Astrophys. J. (2011).

[21] Hawking, S. W., "Black hole explosions?," Nature 248, 30-31 (Mar. 1974) DOI: 10.1038/248030a0 .

[22] Rees, M. J., "A better way of searching for black-hole explosions," Nature 266, 333-334 (Mar. 1977) DOI:10.1038/266333a0.

[23] Cognard, I., Shrauner, J. A., Taylor, J. H., and Thorsett, S. E., "Giant Radio Pulses from a Millisecond Pulsar," Astrophys. J. 457, L81-L84 (Feb. 1996) DOI: 10.1086/309894 .

[24] von Korff, J. S., Astropulse: A search for microsecond transient radio signals using distributed computing, $\mathrm{PhD}$ thesis, University of California, Berkeley, CA, USA (May 2010) ADS: 2010PhDT. .......18V .

[25] von Korff, J., Demorest, P., Heien, E., Korpela, E., Werthimer, D., Cobb, J., Lebofsky, M., Anderson, D., Bankay, B., and Siemion, A., "Astropulse: A Search for Microsecond Transient Radio Signals Using Distributed Computing. I. Methodology," submitted to Astrophys. J. (2011).

[26] Kalberla, P. M. W., Burton, W. B., Hartmann, D., Arnal, E. M., Bajaja, E., Morras, R., and Pöppel, W. G. L., "The Leiden/Argentine/Bonn (LAB) Survey of Galactic HI. Final data release of the combined LDS and IAR surveys with improved stray-radiation corrections," Astron. Astrophys. 440, 775-782 (Sept. 2005) DOI:10.1051/0004-6361:20041864 arXiv:astro-ph/0504140. 